\title{
Schlafstörungen bei Kindern - der Stellenwert der Polysomnografie
}

\section{Sleep Disturbances in Childhood - The Rank of Polysomnography}

H. M. Straßburg

Bibliografie

DOI http://dx.doi.org/

$10.1055 / \mathrm{s}-0031-1295467$

Klin Padiatr 2011; 223: 405-407

(c) Georg Thieme Verlag KG

Stuttgart · New York

ISSN 0300-8630

Korrespondenzadresse

Prof. Dr. Hans Michael

\section{Straßburg}

Universitäts-Kinderklinik Josef-Schneider-Straße 2 97080 Würzburg

Tel.: $+49 / 931 / 20127510$

Fax: +49/931/201 27858

strassburg@mail.uni-wuerz

burg.de
Die Schlafmedizin bei Kindern und Jugendlichen hat in den vergangenen 20 Jahren sowohl in der Forschung als auch in der praktischen Umsetzung enorm an Bedeutung gewonnen. Trotz vieler Erkenntnisse und immer neuen Beschreibungen von schlafassoziierten Phänomenen gibt es aber noch viele offene Fragen [14-16]. Deshalb soll der Versuch einer kurzen Bestandsaufnahme vorgenommen werden.

Hierbei wird Gewicht auf die Fragen gelegt, wann eine Polysomnografie nach dem aktuellen Stand der wissenschaftlich begründeten technischen Möglichkeiten zu erfolgen hat bzw. bei welchen Konstellationen aufgrund einer sorgfältig erhobenen Anamnese und der ärztlichen Untersuchung eine Diagnose gestellt und ohne Polysomnografie therapeutische Maßnahmen eingeleitet werden können.

Wir wissen heute, dass es sich beim Schlaf einerseits um den Ausdruck von zirkadianen Prozessen handelt, die vom Nucleus suprachiasmaticus gesteuert werden und andererseits vom Phänomen der Schlafhomöostase. Ob letztere mehr metabolisch oder mehr neuronal reguliert wird, ist immer noch unklar - zweifelsohne spielt sie aber für Gedächtnis- und Lernprozesse eine wesentliche Rolle $[14,15]$.

Der individuelle Schlafbedarf ist sehr unterschiedlich und wird bei Säuglingen mit 12-20 Stunden, bei Erwachsenen mit 4-10 Stunden angegeben, sodass eine große individuelle Variabilität besteht. Auch das Schlafmuster mit den verschiedenen Stadien des orthodoxen Schlafes und dem paradoxen REM-Schlaf zeigt individuell und in Abhängigkeit vom Lebensalter große Unterschiede $[15,16]$.

Mehrere Fragebogen-basierte Studien haben bei $30 \%$ der Kinder und Jugendlichen Störungen des Ein- und oder Durchschlafens festgestellt [14]. Es ist eine wichtige ärztliche Herausforderung, zuerst einmal zwischen nicht-organisch und organisch bedingten Schlafstörungen $\mathrm{zu}$ unterscheiden. Dabei sind eine Vielzahl völlig unterschiedlicher Organsysteme kritisch zu berücksichtigen, vor allem Obstruktionen im Bereich der oberen Luftwege und unterschiedliche Hirnfunktionsstörungen wie Epilepsien oder Hirnstammanomalien (z.B. Chiari-Malformationen) (s.S.429-433), aber auch arterieller Hochdruck, Stoffwechselstörungen, toxische Einflüsse (s.S.408-413), Muskelerkrankungen, Adipositas (s.S.449-453), gastroösophagealer Reflux, Herzinsuffizienz, restriktive Lungenerkrankungen und Hautaffektionen bis hin zu Narkolepsien und primären Atemantriebsstörungen $[4,8,9,11,13]$. Dieses breite Spektrum ganz unterschiedlicher Ursachen weist auf die
Bedeutung - wie bei jeder Diagnosestellung - einer sorgfältig erhobenen Anamnese und der ärztlichen Untersuchung hin.

In der Praxis ist das wichtigste Instrument zur primären Zuordnung einer Schlafstörung die ausführliche Anamneseerhebung in Verbindung mit der Erstellung eines differenzierten SchlafWach-Protokolls über mindestens 2 Wochen $[8,14,15]$.

Nach der International Classification of Sleep Disorders (ICSD-2) von 2005 sind in der frühen Kindheit primäre Schlafapnoen von obstruktiven Schlafapnoe-Syndromen zu differenzieren [1]. Hierbei spielen vor allem Hyperplasien des lymphatischen Rachenringes eine wesentliche Rolle. Es sind aber auch verschiedene kraniofaziale Fehlbildungen mit und ohne Mikrognathie und Zungenanomalien zu erkennen, Hierzu zählen, z.B. das Pierre-Robin-Syndrom und syndromale Kraniostenosen mit knöchernen Choanalanomalien (s.S. 429-433), Fehlfunktionen im Kehlkopf, in der Trachea und im Bronchialbereich wie z.B. Anomalien der großen Mediastinalgefäße und verschiedene oropharyngeale Dysfunktionen, wie sie z.B. bei der Trisomie 21 vorkommen. Primäre Atemantriebsstörungen wie beim UndineSyndrom oder schlafassoziierte Atemstörungen bei einer Herzinsuffizienz erfördern dabei eine besondere diagnostische Sorgfalt dar $[4,12,13]$. Wegen des erheblichen technischen und personellen Aufwandes bei einer vollständigen Polysomnografie ist in der Praxis ein stufenweises Vorgehen bei Verdacht auf schlafassoziierte Gesundheitsstörungen indiziert $[7,20]$. Dabei werden nach Anamnese und klinischer Untersuchung Kombinationen von Herz-, Atmungs- und pulsoxymetrischer Registrierung des Nachtschlafes eingesetzt. Auf die technischen Besonderheiten bei Säuglingen und Kleinkindern ist dabei naturgemäß besonders zu achten, sodass solche Ableitungen sinnvoller Weise bereits im Rahmen eines ersten stationären Aufenthaltes in einer Klinik stattfinden.

Liegen komplexe Fragestellungen mit erheblichen therapeutischen Konsequenzen vor, sind in einem anerkannten pädiatrischen Schlaflabor komplette Polysomnografien durchzuführen. Hierzu gehören in jedem Fall die Ableitungen von

- thorakalen und abdominalen Atembewegungen

- des oronasalen Atemflusses

- von EKG und Pulsfrequenz

- die transkutane $0_{2}$-Sättigung mittels Pulsoxymetrie

- mindestens 2 technisch einwandfreie EEGAbleitungen 
- das Elektrookulogramm und

- ein EMG der Kinnmuskulatur.

Darüber hinaus ist, je nach Fragestellung, die Registrierung des

- EMGs des M. tibialis anterior,

- von Körperbewegungen,

- die ösophageale pH-Metrie

- die Near-infrared-Spectroscopy der zerebralen Durchblutung

- die transkutane $\mathrm{CO}_{2}$-Messung und

- die Verhaltensbeobachtung, z.B. von Schwitzen, Einziehungen und Anfallssymptomen, mit einer Infrarot-Videokamera notwendig $[1,2,7]$.

Zusätzlich sind z.B. bei Verdacht auf neuromuskuläre Atemstörungen auch kapilläre $\mathrm{CO}_{2}$-Messungen im Nachtschlaf vorzunehmen. Ob eine Nachtschlafregistrierung ausreicht oder ob immer 2 durchgeführt werden sollten, wird kontrovers diskutiert. Letztlich sollten aber bei differenzierten Fragestellungen immer 2 Nachtschlafregistrierungen vorliegen [17,24]. Bei sehr unruhigen Patienten haben sich in jüngster Zeit drahtlose Techniken mit WLAN und Blue tooth bewährt.

Die therapeutischen Konsequenzen können äußerst vielfältig sein: die häufigen HNO-ärztlichen Operationen wie eine Adenotonsillotomie bzw. -ektomie bedürfen in der Regel keiner vorausgehenden stationären Polysomnografie; hier reichen die klinischen Befunde und ambulante Messungen aus [8,20,26]. Umfangreichere plastische Operationen im HNO- und Kieferchirurgiebereich, neurochirurgische Operationen, z. B auch im Bereich des Foramen magnum, oder Entlastungen des intrakraniellen Druckes erfordern hingegen eine sehr genaue Indikation und sollten mit differenzierten polysomnografischen Befunden überprüft werden (s.S.429-433) [2,12,20,26]. Jenseits der frühen Säuglingszeit werden atemstimulierende Medikamente nur noch selten empfohlen, hier kommen vor allem die verschiedenen Formen der nächtlichen Atemunterstützung mit CPAP und BiPAP infrage [11,12].

Ein besonders schwieriges Thema stellt die Indikation zur Polysomnografie bei evtl. nur von den Eltern beobachteten Atemstörungen dar, z.B. im Sinne eines ALTE-Ereignisses oder bei einem plötzlichen Kindstod von anderen Familienmitgliedern, z. B. einem Geschwisterkind $[2,8,22,23]$. Einerseits sind die Eltern in ihrem Bestreben zu unterstützen, jede nur mögliche Vorsorge zu treffen. Andererseits haben umfangreiche wissenschaftliche Untersuchungen zu dieser Thematik bis heute keinen gesicherten Zusammenhang zwischen Befunden der klassischen Polysomnografie und dem objektivierbaren Risiko eines SIDS ergeben, wohl aber unterschiedliche andere Befunde wie vegetative Symptome und Bewegungsanomalien im Schlaf, die ein möglicher Indikator für ein erhöhtes Risiko darstellen [5, 6, 10,23].

Dennoch werden immer wieder falsche Analogieschlüsse aus polysonografischen Untersuchungen und Verunsicherungen als Begründung für die Verordnung unterschiedlicher Heimmonitore und anderer Überwachungsgeräte herangezogen $[5,7,10,23]$. Dies ist neben den erheblichen Kosten vor allem auch mit nicht akzeptablen psychischen Belastungen für die betroffenen Familien, z.B. infolge der ständigen Fehlalarme, verbunden und hält zudem in aller Regel einer kritischen Belastungs-Nutzen-Überprüfung nicht stand $[20,23]$.

Neben der Indikationsstellung zu solchen Untersuchungen ist es aber die Aufgabe eines jeden Kinderarztes in Praxis und Klinik, immer wieder auf die gesicherten präventiven Maßnahmen zur Schlafensverbesserung (s. S. 408-413) bzw. zur Vermeidung von Gefährdungen im Schlaf bei jungen Kindern hinzuweisen $[3,9,18,19,21,22]$. Nach wie vor gehören hierzu vor allem:
- der absolute Verzicht des Rauchens der Mutter während der Schwangerschaft und in der Stillzeit

- eine konsequent rauchfreie Umgebung des Kindes

- der absolute Verzicht auf Alkohol und die Vermeidung von ZNS-wirksamen Medikamenten in Schwangerschaft und Stillzeit

- die richtige Schlafposition in Rückenlage unter möglichster Vermeidung von Lagerungshilfen und Kopfkissen auf einer nicht zu weichen Matratze mit einem Schlafsack

- die Vermeidung von Schnüren u.ä. im Bett

- die Vermeidung von Überwärmung

- regelmäßiges Stillen, möglichst ohne Zufütterung

- die Vermeidung von potenziell gefährlicher Zusatznahrung (Botulismus!) und

- evtl. die Verwendung eines adäquaten Schnullers.

Wenn bei einem zuvor unauffällig schlafenden Kind akut im Schlaf Schreizustände und andere Verhaltensauffälligkeiten beobachtet werden, ist immer an organische Ursachen (Invagination, Hirnturmoren u.v.m.) (s.S. 419-423) zu denken [19,25].

Aktuelle Erkenntnisse in der Schlafforschung bei Erwachsenen mit modernen Techniken, z.B. der funktionellen Kernspintomografie, der Positronen-Emissions-Tomografie und neuen elektrophysiologischen Ableitetechniken, bedürfen der sorgfältigen und kritischen Überprüfung sowie Validierung in Abhängigkeit vom Alter bzw. dem Entwicklungsstand, um ihre Bedeutung für ein besseres Verständnis des kindlichen Schlafes zu erfassen $[14,15]$.

Interessenkonflikt: Die Autoren erklären hiermit, dass kein Interessenkonflikt besteht.

\section{Literatur}

1 American Academy of Sleep Medicine (AASM). ICSD-2: International Classification of sleep disorders: diagnostic and coding manual. Westchester, IL, 2005

2 Aurora RN, Zak RS, Karippot A et al. Practice parameters for the respiratory indications for polysomnography in children. Sleep 2011; 34: $379-388$

3 Bartram U, Singer D. Säuglingsbotulismus und plötzlicher Kindstod: Eine kritische Bestandsaufnahme. Klin Padiatr 2004; 216: 26-30

4 Demir Z, Böckelmann S, Kutschke $G$ et al. Ungewöhnlich milder Verlauf eines Undine-Syndroms bei einem reifen Neugeborenen und familiäre Häufung. Klin Padiatr 2010; 222 (Suppl 1): S98. DOI:10.1055/s-0030-1261599

5 Edner A, Wennborg M, Alm B et al. Why do ALTE infants not die in SIDS? Acta Paediatr 2007; 96: 191-194

6 Einspieler C, Prechtl HF, van Eykern L et al. Observation of movements during sleep in ALTE (apparent life threatening event) and apnoeic infants - a pilot study. Early Hum Dev 1994; 40: 39-49

7 Erler T, Oehlschläger J, Nowotny TJ et al. Polysomnographie im Säuglingsalter - notwendig oder teurer Luxus? Therapeutische und interventionelle Konsequenzen. Klin Padiatr 2001; 213: 114-121

8 Erler T, Paditz E. Obstructive sleep apnea syndrome in children: a state-of-the-art review. Treat Respir Med 2004; 3: 107-122

9 Fischer D, Freislederer A, Jorch G. Plötzlicher Tod bei Zwillingen. Botulismus durch Kontaminations- und Gemüsebrei. Klin Padiatr 2004; 216: $31-35$

10 Franco $P$, Montemitro E, Scaillet $S$ et al. Fewer spontaneous arousals in infants with apparent life-threating event. Sleep 2011; 34: 733-743

11 Flechtner-Mors M, Thamm M, Rosario AS et al. Hypertonie, Dyslipoproteinämie und BMI-Kategorie charakterisieren das kardiovaskuläre Risiko bei übergewichtigen oder adipösen Kindern und Jugendlichen: Daten der BZgA-Beobachtungsstudie (EvAKuJ-Projekt) und der KiGGSStudie. Klin Padiatr 2011; 223: 449-453

12 Hein A, Schweitzer T, Straßburg HM et al. Diagnostik und Therapie des obstruktiven Schlafapnoe-Syndroms bei Kindern mit syndromalen prämaturen Kraniosynostosen. Klin Padiatr 2011; 223: 429-433

13 Heine $K$, Urschitz $M$, Poets $C$. Herzinsuffizienz als Manifestation eines obstruktiven Schlaf-Apnoe-Syndroms. Klin Padiatr 2010; 222: 90-91 
14 Jenni OG, Benz C. Schlafstörungen. Pädiatrie up2date 2007; 4: 309-333

15 Jenni OG, Carskadon MA, Hrsg. Sleep medicine clinics: Children and adolescents. Philadelphia PA: Elsevier-Sciene, 2007

16 Jenni OG, Molinari L, Caflisch JA et al. Sleep duration from age one to ten years: Variability and stability in comparison with growth. Pediatrics 2007; 120: 769-776

17 Katz ES, Greene MG, Carson KA et al. Night-to-night variability of polysomnography in children with suspected obstructive sleep apneas. J Pediatr 2002; 140: 589-594

18 Kelmanson IA, Erman LV, Litvina SV. Maternal smoking during pregnancy and behavioural characteristics in 2-4 month old infants. Klin Padiatr 2002; 214: 359-364

19 Kelmanson IA. Pacifier use and sleep position in 2 to 4 month old infants. Klin Padiatr 2000; 212: 273-276

20 Kuhle S, Urschitz MS, Eitner S et al. Interventions for obstructive sleep apnea in children: a systematic review. Sleep Med Rev 2009; 13: 123-131
21 Müller MJ, Lange M Paul T et al. Stillen unter Methadon - bzw. Buprenorphin-Substitutionstherapie. Klin Padiatr 2011; 223: 408-413

22 Nennstiel-Ratzel U, Hölscher G, Ehrensperger-Reeh P et al. Prevention of Sudden Infant Death Syndrome (SIDS) in Bavaria - evaluation of the prevention campagne. Klin Padiatr 2010; 222: 45-50

23 Poets CF. Apnea of prematurity: What can observational studies tell us about pathophysiology? Sleep Med 2010; 11: 701-707

24 Verhulst SL, Schrauwen N, De Backer WA et al. First night effect for polysomnographic data in children and adolescents with suspected sleep disordered breathing. Arch Dis Childh 2006; 91: 233-237

25 Weiß F, Streng A, von Kries R et al. Incidence of intussusception in early infancy: A capture - recapture estimate for Germany. Klin Padiatr 2011; 223: 419-423 (301)

26 Wise MS, Nichols CD, Grigg-Damberger MM et al. Executive summary of respiratory indications for polysomnography in children: an evidencebased review. Sleep 2011; 34: 389-398 